\title{
Diagramme d'équilibre liquide vapeur du système binaire $\mathrm{NH}_{3}-\mathrm{CH}_{3} \mathrm{NH}_{2}$
}

\author{
R. Bellajrou, M.Th. Cohen-Adad ${ }^{1}$ et M. El Hadek \\ Laboratoire de Chimie Minérale Appliquée et Génie des Procédés, Université Ibn Zohr, \\ BP. 28/S, Agadir, Morocco \\ ${ }^{1}$ Laboratoire de Physico-Chimie des Matériaux Luminescents, Université Claude Bernard \\ Lyon I, 69622 Villeurbanne cedex, France
}

\begin{abstract}
A static equilibrium apparatus is described which permits vapour - liquid equilibrium measurements in the range of 293 to $473 \mathrm{~K}$ and pressure up to $100 \mathrm{bar}$. Both phases are analysed in a gas chromatography. Measurements are reported on ammonia - monomethylamine. The temperature - composition phase diagram of the binary system between 6 to $18 \mathrm{bar}$ is given.
\end{abstract}

\section{INTRODUCTION}

L'élaboration de la monométhylhydrazine $\mathrm{CH}_{3} \mathrm{NHNH}_{2}$ connue sous le sigle $\mathrm{MMH}$, par le procédé Raschig conduit à des solutions de synthèse très diluées, constituées essentiellement de $\mathrm{H}_{2} \mathrm{O}, \mathrm{CH}_{3} \mathrm{NH}_{2}$, $\mathrm{NH}_{3}, \mathrm{NaCl}$ et $\mathrm{NaOH}[1,2]$. La première étape, des opérations de traitement de cette solution, correspond à une distillation afin d'éliminer les constituants les plus volatils $\mathrm{CH}_{3} \mathrm{NH}_{2}$ et $\mathrm{NH}_{3}$. Après condensation du distillat, ces amines sont ensuite séparées en vue de leur recyclage dans le procédé de synthèse. La détermination des conditions de séparation a nécessité l'étude préalable de l'équilibre liquide - vapeur du système binaire $\mathrm{NH}_{3}-\mathrm{CH}_{3} \mathrm{NH}_{2}$.

\section{EXPÉRIENCES MISES EN GEUVR}

Le dispositif expérimental mis au point pour cette étude a été construit autour d'un réacteur de $100 \mathrm{ml}$ en acier inoxydable (304) compatible avec les amines utilisées [3] (figure 1). La température est mesurée au moyen d'un thermocouple chromel-alumel $(\mathrm{NiCr}-\mathrm{Ni})$ associé à un lecteur numérique (AOIP). Et la pression est indiquée par un conditionneur lecteur CD2000 couplé à un capteur de pression couche mince type P201 A30 (Essor Français Electronique). L'appareillage construit pour cette étude permet d'opérer dans un domaine de température comprise entre 293 et $473 \mathrm{~K}$, et jusqu'à 100 bars pression.

Nous avons utilisé l'ammoniac pur anhydre à $99,96 \%$ (fourni par la société Air Liquide) et la monométhylamine employée titre $97,0 \%$ (produit Merck).

Le protocole opératoire adopté pour la conduite d'une mesure est le suivant :

L'ensemble de l'appareillage préalablement purgé à l'azote et maintenu sous vide. Le réacteur de mesure plongé à mi-hauteur dans l'air liquide, l'ammoniac puis la monométhylamine sont introduits au moyen de la vanne a. Ensuite, l'autoclave est isolée (vanne b et $c$ fermées) et plongée dans un bain thermostatique, la pression est alors ajustée par réglage de la température du bain.

Des prélèvements de la phase liquide et de la phase vapeur en équilibre sont effectués pour analyse $\mathrm{CPG}$, en vue de suivre l'évolution de la composition des phases. Le chromatographe utilisé est un appareil HEWLETT-PACKARD 5890A couplé à un intégrateur numérique 3393A. La séparation est réalisée au moyen d'une colonne garnie de Tenax (plymère poreux à base d'oxyde 2,6 diphenyl paraphenylène, (produit AKZO). Le gaz vecteur employé est l'Hélium U. 


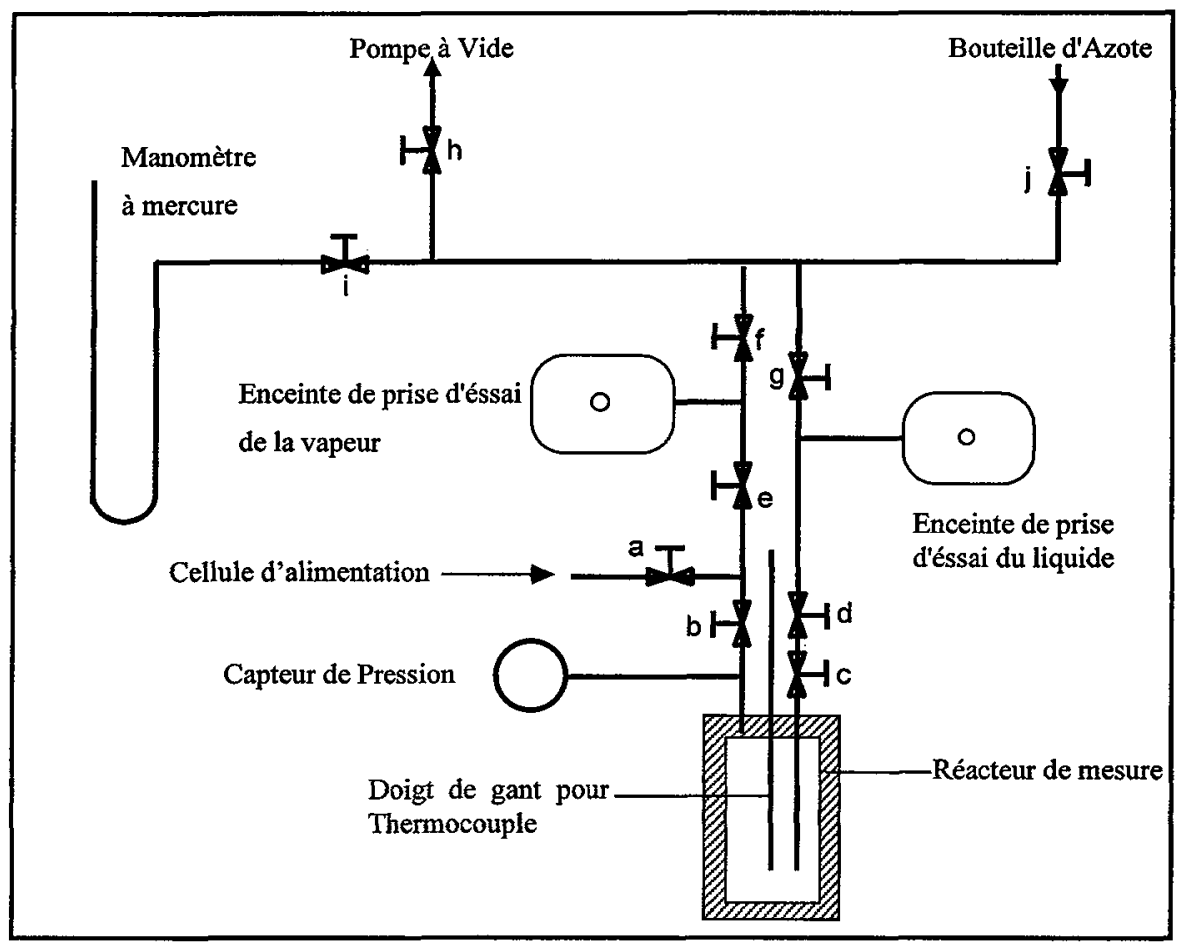

Figure 1. Schéma du dispositif expérimental

\section{EXPLOITATION DES RÉSULTATS}

Pour une série de mélanges de compositions définies, la composition des phases liquide et vapeur en équilibre a été déterminée à différentes températures et sous pression. Pour chaque mélange, les résultats obtenus ont été exprimés sous forme de courbes traduisant l'évolution de la pression et de la composition des phases liquide et vapeur en fonction de la température. Il est ensuite possible par simple interpolation de déduire, pour une isobare choisie, la température et la composition des phases en équilibre.

La figure 2 présente les diagrammes isobares des équilibres liquide - vapeur dans le système $\mathrm{NH}_{3}$ $\mathrm{CH}_{3} \mathrm{NH}_{2}$ obtenus à $6,10,14$ et 18 bars.

Il est à noter que les températures inférieures à l'ambiante ont été obtenues par extrapolation.

\section{INTERPRÉTATIONS DES COURBES ET CONCLUSION}

L'examen des courbes tracées fait apparaître aussitôt :

- qu'il ne se forme pas de mélange azéotropique dans ce binaire

- que les courbes expérimentales obtenues présentent bien une allure régulière

- que le mélange $\mathrm{NH}_{3}-\mathrm{CH}_{3} \mathrm{NH}_{2}$ ne se comporte pas d'une manière idéale

- que si l'on observe une déformation de la lentille d'équilibre, par rapport au diagramme calculé dans le cadre d'un comportement idéal, cette déformation elle-même est régulière.

Bien que les mélanges $\mathrm{NH}_{3}-\mathrm{CH}_{3} \mathrm{NH}_{2}$ présentent un écart à l'idéalité, les deux courbes de rosée et d'ébullition sont assez écartées l'une de l'autre. Il en résulte que la séparation des deux composés par distillation sera particulièrement aisée [5]. 




Figure 2. Equilibres liquide - vapeur du système $\mathrm{CH}_{3} \mathrm{NH}_{2}-\mathrm{NH}_{3}(-)$ diagramme expérimental (---) diagramme idéal

\section{Références}

[1] Abraham R., Aubry B., Cohen-Adad M. Th. et Cohen-Adad R., Brevet 8409738 ext. Europ. 2566385

[2] Giudice M., Thèse de doctorat. 54 -89 Lyon (1989)

[3] Bellajrou R., Thèse de doctorat. 30 - 92 Lyon (1992)

[4] Bellajrou R., Abraham R., Cohen-Adad M. Th., Cohen-Adad R., XV JEEP Grenoble (1989)

[5] Pannetier G., Mignotte P., Bull. Soc. Chim. Fr. (1963) pp.694-698 